\title{
Estilos de cuidado y la implicación como protector psicológico del cuidador de personas con Parkinson en España
}

\section{Care styles and involvement as a psychological protective of the caregiver of}

\author{
people with Parkinson's disease in Spain
}

\author{
Antonio Aguilar Agudo ${ }^{1}$, Javier Herruzo Cabrera ${ }^{2}$ y María José Pino Osuna ${ }^{3}$
}

${ }^{1}$ Doctor en Psicología. Director de la Asociación de Parkinson de Córdoba (España).

Investigador del grupo de investigación: "Comportamiento de riesgo, salud y seguridad laboral", Departamento de Psicología, Universidad de Córdoba (España). E-mail: z22aguaa@gmail.com

${ }^{2}$ Doctor en Psicología. Catedrático de la Universidad de Córdoba (España), Departamento de

Psicología, Área de conocimiento, personalidad, evaluación y tratamiento psicológicos.

E-mail: ed1hecaf@uco.es

${ }^{3}$ Doctora en Psicología. Catedrático de la Universidad de Córdoba (España), Departamento de

Psicología, Área de conocimiento, personalidad, evaluación y tratamiento psicológicos.

E-mail: ed1piosm@uco.es

Asociación de Parkinson de Córdoba, España.

Universidad de Córdoba, España.

\section{Resumen}

Los familiares de personas con enfermedad de Parkinson (EP) se enfrentan a una variedad de situaciones estresantes como consecuencia del desempeño de su rol de cuidadores. Esto puede tener un impacto negativo en su calidad de vida. Un mayor conocimiento de la relación entre las variables evaluadas en los cuidadores puede ser importante de cara a implementar programas de intervención eficaces. El objetivo fue explorar la personalidad y las estrategias de afrontamiento en familiares cuidadores de personas con EP, estudiando su relación con el malestar psicológico y el grado de implicación en los cuidados. Se trabajó con una muestra de 106 cuidadores que completaron los cuestionarios de personalidad NEO-FFI, estrategias de afrontamiento COPE-28, malestar psicológico CORE-OM y un instrumento para evaluar la implicación del cuidador realizado a partir del índice Katz. El análisis de regresión logística mostró que las personas con puntuaciones por encima de la media en neuroticismo y por debajo en el resto de factores de personalidad y estrategias de afrontamiento tenían más riesgo de padecer malestar psicológico $(\mathrm{OR}=9.250 ; \mathrm{p}<.001) \mathrm{y}$ una menor implicación en los cuidados (OR $=4.125 ; \mathrm{p}<.001)$. Estos resultados sugieren que la personalidad y las estrategias de afrontamiento pueden contribuir a identificar estilos para los cuidados más eficientes, que impliquen una mayor participación en los cuidados con un menor impacto negativo.

Palabras clave: enfermedad de Parkinson, cuidadores, malestar psicológico, personalidad, estrategias de afrontamiento.

\section{Abstract}

Carers of people with Parkinson's disease (PD) experience stressful situations as a result of performing their role as caregivers. This can have a negative outcome on their quality of life. A greater understanding of the rela- 
tionship between the variables assessed in caregivers can be important in implementing effective intervention programs. The aim of this study was to explore the personality and coping strategies used by family caregivers of people with $\mathrm{PD}$, analyzing their relationship with psychological distress and the carer involvement. A cross-sectional study based on a sample of 106 caregivers of people with Parkinson's disease tested the hypothesis that personality and coping strategies can contribute to identifying differences in carer involvement and psychological distress. Caregiver psychological distress was assessed using the CORE-OM questionnaire. The CORE-OM is able to distinguish the population within the clinical and non-clinical range. NEO Five-Factor Inventory (NEOFFI) was used to assess personality. Coping strategies were evaluated with the COPE-28 inventory. An operational measure of the carer involvement was also used. For this purpose, an adaptation of the Katz index assessing basic functional capacity was made. Results: First, all cases were clustered according to personality variables and coping strategies. A K-means cluster analysis was performed and two groups were requested. After this, binary logistic regression analysis was used to test the research hypothesis. The resulting clusters in the above analysis were selected as predictors. Two analyses were performed. In the first, the dependent variable in logistic regression analysis was the psychological distress of the caregivers (dichotomized variable: clinical and non-clinical). Results showed that people clustered within cluster 1 had a 9.250 times higher risk of psychological distress than those included in cluster $2(\mathrm{OR}=9.250 ; \mathrm{p}<$ $.001)$. In a second logistic regression analysis, the dependent variable was participation in care (dichotomized variable: low - high carer involvement). The group of people belonging to cluster 1 showed a carer involvement 4.125 times lower than the people grouped in cluster $2(\mathrm{OR}=4.125 ; \mathrm{p}<.001)$. The results of this research suggest the existence of a relation- ship between psychological variables and carer involvement, showing two different styles of care, which are described based on the personality and coping strategies of the family caregivers. In addition, we found that these styles of care show clear differences in efficiency, reflected in greater carer involvement and a lower psychological distress. The results reported that the cluster of people with below-average scores on neuroticism and above-average scores on the other personality factors (extraversion, openness to experience, agreeableness, and conscientiousness) and coping strategies (planning; active coping; emotional support; social support; positive re-evaluation and acceptance) were associated with increased carer involvement and reduced risk of psychological distress. It has already been suggested that high scores in neuroticism may affect the need to take on a large number of care-related tasks or manage stressful situations in people with PD (Tew et al., 2013). However, our results indicate that the definition of different types of caregivers, where other care-related variables are also included, can help define more efficient styles of care. This is associated with increased carer involvement and minor psychological distress. We propose that in the future these variables be investigated in order to know the outcome of carer involvement and psychological distress, contributing to the description of more efficient care styles in family caregivers. Keywords: Parkinson's disease, caregivers, psychological distress, personality, coping strategies.

\section{Introducción}

Las enfermedades crónicas son una de las principales causas que conducen a las personas a un estado de dependencia. Esto está motivado por la especial dificultad en el abordaje clínico y asistencial en los pacientes. La dependencia surge a partir de un déficit o una disfunción en el organismo que provoca una serie de limitaciones. Esta dificultad no es 
solventada con una adaptación personal o del entorno, sino que precisa de la ayuda de otras personas para el normal desarrollo de las actividades de la vida diaria (AVD) (Gómez-Jarabo y Peñalver, 2007).

Una parte de las personas en situación de dependencia tienen diagnosticada alguna enfermedad neurodegenerativa. Estas se caracterizan por la destrucción o disfunción progresiva de células nerviosas en diferentes zonas del cerebro, lo cual provoca la aparición de signos y síntomas característicos. La Enfermedad de Parkinson (EP) es un trastorno neurodegenerativo que afecta a millones de personas. Existe una variabilidad en la frecuencia de casos (prevalencia e incidencia): es mayor en Europa y EE.UU. que en el resto del mundo (Benito-León, 2018). En las últimas décadas el número de diagnósticos se ha duplicado, y ha pasado de 2.5 millones en 1990 a 6.1 millones el 2016 (Dorsey et al., 2018). La prevalencia se estima en $0.3 \%$ de la población general y el $1 \%$ en mayores de 60 años. La incidencia es de 8 a 18 por $10^{5}$ habitantes/año (Tysnes y Storstein, 2017). En España, la prevalencia media de EP es de 682.2 por $10^{5}$ habitantes/año y una incidencia de 8.2 por $10^{5}$ habitantes/año (García-Ramos, Valdés, Ballesteros, Jesús y Mir, 2016).

La EP es una enfermedad crónica que progresa de forma diferente, dependiendo del individuo, y el envejecimiento y el sexo masculino son los mayores factores de riesgo (Benito-León, 2018). En el caso de los hombres se observa una mayor incidencia entre los 70-74 años y, en las mujeres, a los 85 años (García-Ramos, Valdés, Ballesteros, Jesús y Mir, 2016). En cuanto a la tasa de mortalidad, se han encontrado diferencias: los niveles son mayores para el sexo masculino, las personas de raza blanca y con edades más avanzadas (Benito-León, Louis, Villarejo-Galende, Romero y Bermejo-Pareja, 2014).

La EP se asocia a una pérdida de la autonomía personal y una consecuente demanda de ayuda externa. Se establece entonces una relación de cuidados dentro del ámbito fami- liar, en la que se proporcionan apoyos para el desarrollo de las AVD. Las personas con EP suelen requerir en un primer momento la colaboración de los familiares para actividades puntuales, y esto se convierte progresivamente en una ayuda continuada. Esta situación requiere asimilar los cambios (físicos y mentales) que se producen en la persona que recibe los cuidados, asumir un número elevado de tareas dentro y fuera del hogar, realizar cambios en el proyecto de vida personal, un mayor aislamiento social o tener dificultades financieras (Espinosa-Miranda y Jofre-Aravena, 2012). Esta situación provoca un deterioro de las herramientas personales necesarias para afrontar las situaciones adversas (Espinosa, Guzmán, Bautista y Ramos, 2018).

Los cuidados se basan en una relación no profesionalizada de prestación de servicios y tienen como característica la de constituir un sistema privado en el que la atención se reparte entre familiares, amigos u otras personas allegadas (Jofré y Mendoza, 2005). Tradicionalmente, los cuidados han recaído sobre las mujeres (Cueto y Martínez, 2017; Garzón Patterson, Pascual Cuesta y Collazo Lemus, 2018; Giraldo, Zuluaga y Uribe, 2018; Reyes y Muñoz-Quezada, 2019), y llegan a alcanzar hasta un $75 \%$ en la EP (Sturm, Folkerts y Kalbe, 2018). Tales diferencias de sexo han sido atribuidas a la divergencia en los modelos de crianza, sugiriéndose niveles más elevados de empatía en las mujeres (Paez y Rovella, 2019). Sin embargo, en este caso es importante destacar que la EP no afecta por igual a ambos sexos; es más común su aparición en los hombres (Benito-León, 2018) y es el cónyuge quien, en mayor medida, se ocupa de los cuidados (Martín, 2016).

Los cuidados continuados a personas con EP pueden tener un impacto negativo en el entorno familiar, que pueden llegar a experimentar malestar psicológico como consecuencia de su labor (Blanco Buitrago, Martínez Wilches, Velasco Malaver, Wilches Najar y Bernal Jiménez, 2017). Esto puede condicionar la calidad de vida de las personas 
que reciben los cuidados (Cueto y Martínez, 2017).

El malestar psicológico comprende una serie de manifestaciones clínicas no patológicas y arraigadas en el carácter de la persona (Hernández et al., 2006). Puede estar provocado por algunos factores psicológicos, entre ellos se encuentran ciertos patrones de afrontamiento a situaciones estresantes (Baltar, Cerrato, de Trocóniz y Márquez-González, 2006) o las creencias irracionales (Stebbins y Pakenham, 2001). Existe evidencia de mejora del malestar psicológico a partir del desarrollo de programas de intervención basados en el manejo de las emociones o bien a través de la reducción de las situaciones estresantes relacionadas con los cuidados (Baltar, Cerrato, de Trocóniz y Márquez-González, 2006).

Varios estudios han descrito los efectos negativos a los que se enfrentan las personas como consecuencia de asumir los cuidados de manera continuada. El malestar psicológico puede mostrar diferencias en las personas respecto a la capacidad de adaptarse a este tipo de situaciones. Dentro del ámbito de los cuidados, se enfrentan un conjunto de situaciones, y existen diferencias individuales en cuanto a la elección de unas u otras alternativas de respuestas. La personalidad y las estrategias de afrontamiento pueden ayudar a explicar tales diferencias.

Las características de la personalidad en personas con la EP han sido investigadas (Santangelo et al., 2018); sin embargo, son escasas las iniciativas que se centraron en la personalidad de los cuidadores. La depresión y la ansiedad son las variables predictivas más importantes para explicar una reducción de la calidad de vida entre los cuidadores. Están asociadas con el factor de personalidad neuroticismo. Los cuidadores que presentan niveles altos de neuroticismo pueden tener más dificultades para asumir un mayor número de tareas o manejar situaciones estresantes (Tew et al., 2013), así como un efecto negativo en la calidad de vida (Ma et al., 2018).

Las estrategias de afrontamiento suelen estar incluidas en investigaciones que abordan los cuidados a personas dependientes. Consisten en acciones específicas de las personas para hacer frente a situaciones valoradas como estresantes (Lazarus, 1993) $\mathrm{y}$, junto a la personalidad, desempeñan una función mediadora en la respuesta del individuo (Cassaretto, 2010).

Existen varias formas de agrupar las distintas dimensiones que componen las estrategias de afrontamiento. Entre ellas está la distinción entre estrategias de tipo adaptativas (afrontamiento activo; planificación; reinterpretación positiva; aceptación; uso de apoyo emocional) y estrategias de tipo desadaptativas (negación; desvinculación comportamental; autodistracción/desvinculación mental; expresión de emociones negativas; religión; uso de sustancias) (Carver, 1997).

Algunos factores como la edad y género del cuidador, la convivencia o el tiempo de cuidado pueden influir en las estrategias de afrontamiento utilizadas (Fernández, Mengíbar, Cano y Leyva, 2016). Por su parte, algunos de los mecanismos de afrontamiento más empleados por los cuidadores de personas con demencia fueron la aceptación, el afrontamiento activo y la religión (León-Campos, Chonchol y Miranda-Castillo, 2018).

La personalidad y las estrategias de afrontamiento están relacionadas entre sí. Reflejan diferencias individuales y son relativamente estables. La personalidad ejerce una influencia sobre la elección de las estrategias de afrontamiento que las personas utilizan para hacer frente a situaciones estresantes; sin embargo, el tipo de respuesta utilizado puede tener consecuencias sobre la salud y el bienestar (Cuéllar Flores y Sánchez López, 2012; Martínez Ortega, 2017).

Los cuidadores se enfrentan a una variedad de situaciones estresantes como consecuencia del desempeño de su rol. Este tipo de situaciones suceden y son heterogéneas, lo que plantea una variabilidad en la forma en que son percibidas y la importancia que se les atribuye. Del mismo modo, existe una varia- 
bilidad en el tipo de respuesta que pueden ser utilizadas ante un mismo hecho, lo cual constituye diferentes estilos para los cuidados. Los estilos de cuidado pueden ser definidos como un patrón habitual de respuestas para satisfacer la demanda (manifiesta o latente) de cuidados. Tanto la personalidad como las estrategias de afrontamiento pueden contribuir a explicar diferencias en los estilos de cuidado, lo cual determina el papel que desempeña el cuidador y la influencia que se ejerce sobre el bienestar de la persona que recibe los cuidados.

Tomando lo anterior como punto de partida, este estudio tiene como finalidad indagar sobre los factores que puedan estar relacionados con la capacidad de adaptación de las personas en respuesta a los cuidados. Por ello, el objetivo consistió en evaluar la personalidad y las estrategias de afrontamiento utilizadas por los cuidadores de personas con EP y analizar su relación tanto con el grado de implicación en los cuidados como con el malestar psicológico. Se plantea así la hipótesis de que la personalidad y las estrategias de afrontamiento pueden contribuir a identificar grupos de cuidadores que muestren diferencias en cuanto a su grado de participación en los cuidados y el malestar psicológico asociado.

\section{Método}

\section{Diseño de investigación}

Estudio transversal basado en datos extraídos sobre variables psicológicas (personalidad, estrategias de afrontamiento y malestar psicológico) y el grado de implicación en los cuidados. La unidad objeto de análisis fue los familiares que se ocupaban de los cuidados a personas con la EP.

\section{Participantes}

Se seleccionó una muestra que coincidió con la asistencia de los pacientes con EP a las consultas. Se obtuvieron datos de 106 familiares cuidadores que acompañaban a la persona con EP, provenientes de una diversidad de fuentes de procedencia hospitalaria y de asociaciones de pacientes en España. El $74 \%$ de cuidadores eran mujeres, con una edad promedio de 58 años $(\mathrm{DE}=14)$. Los criterios de inclusión incluyeron el diagnóstico confirmado de la persona con EP que recibía los cuidados. Se excluyeron personas con dificultades cognitivas que les impidieran comprender el objetivo del estudio, el contenido de los instrumentos y responder a ellos.

\section{Instrumentos}

Para evaluar la personalidad se utilizó el inventario NEO reducido de cinco factores (NEO-FFI), versión española del Revised NEO Personality Inventory. El NEO-FFI ofrece una medida rápida y general de los cinco factores de personalidad: neuroticismo, extraversión, apertura, amabilidad y responsabilidad. Es un instrumento ampliamente utilizado que muestra una alta consistencia interna: alfa de Cronbach comprendidos entre .82 y .90 (Costa y McCrae, 2008).

El malestar psicológico del cuidador se evaluó con el Clinical Outcomes in Routine Evaluation (CORE-OM), versión española. El CORE-OM permite clasificar los casos entre población clínica y no clínica. Está formado por cuatro dimensiones: bienestar subjetivo; problemas/síntomas, (ansiedad, depresión, trauma y síntomas físicos); funcionamiento general, (relaciones íntimas, sociales y otros aspectos del funcionamiento cotidiano) y riesgo (autolesiones o daños a terceros). Satisface los criterios psicométricos de fiabilidad y validez, con una alta consistencia interna y fiabilidad test-retest: alfa de Cronbach entre .75 y .90 y correlación test-retest entre .87 y .91 (Feixas et al., 2012).

Las estrategias de afrontamiento se evaluaron con el COPE-28, que es la versión española del Brief-COPE de Carver (1997). Es un inventario que contiene 14 subescalas entre las que se encuentran: afrontamiento activo, planificación, apoyo instrumental, uso de 
apoyo emocional, autodistracción, desahogo, desconexión conductual, reinterpretación positiva, negación, aceptación, religión, uso de sustancias, humor y autoinculpación. La respuesta a cada ítem es mediante una escala ordinal tipo Likert con cuatro alternativas de respuesta que van desde nunca hago esto hasta siempre hago esto, con puntuaciones intermedias (Morán, Landero y González, 2010).

Se utilizó una medida operativa del grado de implicación en los cuidados. Para ello, se realizó una adaptación del índice de Katz que valora la capacidad funcional básica (Katz et al., 1963). El grado de implicación en los cuidados se evaluó cuantificando el tiempo invertido por los cuidadores en cada una de las AVD: baño, vestido, uso del WC, movilidad, continencia y alimentación.

\section{Procedimiento}

En un primer momento, cada una de las personas recibió información suficientemente clara sobre el contenido del estudio. Se les requirió además su consentimiento para participar, y se garantizó la protección de los datos personales conforme a la legislación vigente. Una vez que las personas aceptaron ser incluidas en el estudio, se procedió a realizar la recogida de datos de manera individual, facilitando para ello un protocolo que incluía los cuestionarios seleccionados. Las pruebas fueron administradas por miembros del equipo de investigación capacitados en la administración de los instrumentos en una única sesión de unos 60 minutos de duración.

El Comité de Ética de la Investigación del Hospital Universitario Reina Sofía de Córdoba (España) aprobó el procedimiento de investigación y certificó que el proyecto cumplía con los principios establecidos por la declaración de Helsinki de 1975, revisada en 2000 y 2008, y los criterios de la Asociación Médica Mundial relacionados con los Derechos Humanos y la Biomedicina, modificados por el Consejo del Convenio Europeo de 1996.

\section{Análisis de datos}

Una vez completados los cuestionarios con la información requerida, se tabularon los datos para ser analizados con el programa de software estadístico PASW Statistics version 18.0.0 (IBM SPSS Statistics). Mediante una técnica multivariante, se agruparon los casos en función de la similitud existente entre personalidad y estrategias de afrontamiento mediante el análisis de conglomerados de K-medias.

A partir de la variable creada con los conglomerados de pertenencia, se realizó un análisis de regresión logística binaria para conocer el grado de implicación en los cuidados como función de la personalidad y las estrategias de afrontamiento y explorar el riesgo de padecer malestar psicológico en cuidadores.

\section{Resultados}

Para probar la hipótesis de que el malestar psicológico y el grado de implicación del cuidador podrían variar en función de la personalidad y las estrategias de afrontamiento, se exploró en la muestra la existencia de perfiles diferenciados de personas en función de estas variables psicológicas. El análisis de conglomerado de K-medias permitió agrupar los casos según las variables de personalidad y las estrategias de afrontamiento. Se solicitaron dos grupos, y se creó una nueva variable a partir del conglomerado de pertenencia. En relación con la personalidad, se incluyeron los cinco factores de personalidad evaluados con el NEO-FFI (neuroticismo, extraversión, responsabilidad, apertura y amabilidad). Con respecto al afrontamiento, se optó por seleccionar en el COPE-28 las dimensiones compatibles con las estrategias de afrontamiento de tipo adaptativas, adoptando para ello la clasificación propuesta por Carver (1997).

La tabla 1 muestra las variables incluidas dentro de cada uno de los conglomerados y sus respectivos valores, expresados en puntuaciones típicas. El primer conglomerado 
está constituido por personas con puntuaciones por encima de la media en neuroticismo (.32703) y puntuaciones por debajo de la media en extraversión (-.18205); apertura (-.32791); amabilidad (-.24649); responsabilidad (-.45840); planificación (-.48746); afrontamiento activo (-.70076); apoyo emocional (-.78927); apoyo social (-.88245); reevaluación positiva (-.64679) y aceptación (-.28279). El segundo conglomerado incluye personas con puntuaciones por debajo de la media en neuroticismo (-.20628) y por encima de la media en extraversión (.11483); apertura (.20684); amabilidad (.15548); responsabilidad (.28914); planificación (.30747); afrontamiento activo (.44202); apoyo emocional (.49785); apoyo social (.55662); reevaluación positiva (.40797) y aceptación (.17837) (Tabla 1).

\section{Tabla 1}

Construcción de los grupos a partir de la personalidad y las estrategias de afrontamiento en cuidadores de personas con EP.

\begin{tabular}{c|c|c|} 
& \multicolumn{2}{|c|}{ Conglomerados } \\
& $\mathbf{1}$ & $\mathbf{2}$ \\
\hline Neuroticismo & .32703 & -.20628 \\
Extraversión & -.18205 & .11483 \\
Apertura & -.32791 & .20684 \\
Amabilidad & -.24649 & .15548 \\
Responsabilidad & -.45840 & .28914 \\
Planificación & -.48746 & .30747 \\
Afrontamiento activo & -.70076 & .44202 \\
Apoyo emocional & -.78927 & .49785 \\
Apoyo social & -.88245 & .55662 \\
Reevaluación positiva & -.64679 & .40797 \\
Aceptación & -.28279 & .17837
\end{tabular}

El análisis de varianza mostró que las diferencias entre los dos conglomerados obtenidos resultaron significativas para todas las variables excepto para el factor de personalidad extraversión $(\mathrm{p}=.137)($ Tabla 2$)$. 
Tabla 2

Tabla ANOVA para cada una de las variables incluidas en los conglomerados obtenidos en la Tabla 1.

\begin{tabular}{c|c|c|} 
& F & Sig. \\
\hline Neuroticismo & 7.600 & .007 \\
Extraversión & 2.242 & .137 \\
Apertura & 7.644 & .007 \\
Amabilidad & 4.186 & .043 \\
Responsabilidad & 16.065 & .000 \\
Planificación & 18.541 & .000 \\
Afrontamiento activo & 47.317 & .000 \\
Apoyo emocional & 68.379 & .000 \\
Apoyo social & 102.298 & .000 \\
Reevaluación positiva & 37.764 & .000 \\
Aceptación & 5.580 & .020
\end{tabular}

El análisis anterior permitió hacer una agrupación de los cuidadores a partir de las puntuaciones obtenidas en la personalidad y las estrategias de afrontamiento. La medición de ambas variables contribuyó a crear sendos grupos con perfiles característicos que sugieren diferentes estilos para los cuidados. Tomando estos datos como predictores, se realizó un análisis de regresión logística binaria. Como variable dependiente se seleccionó el malestar psicológico del cuidador. Para ello, se utilizó la variable dicotomizada en los rangos clínico y no-clínico. Este análisis tenía como propósito tratar de determinar qué estilo de los cuidados predecía un riesgo mayor de padecer malestar psicológico entre los cuidadores.
La Tabla 3 muestra la significación y las odds ratio (OR) correspondientes a los conglomerados 1 y 2 . Como puede observarse, las personas agrupadas dentro del conglomerado 1 (puntuaciones por encima de la media en neuroticismo y por debajo en el resto de variables) tenían un riesgo 9.250 veces mayor de padecer malestar psicológico que las incluidas en el conglomerado 2 (puntuaciones por debajo de la media en neuroticismo y por encima en el resto de variables) $(\mathrm{OR}=$ $9.250 ; p<.001)$. Se aprecia que con un nivel de confianza del $95 \%$, la OR de este factor para el modelo propuesto osciló entre 3.297 y 25.951 por lo que la asociación resultó significativa (el valor 1 no forma parte del intervalo). 
Tabla 3

Modelo predictivo del malestar psicológico a través de una regresión logística.

\begin{tabular}{c|c|c|c|c|c|} 
& & & & \multicolumn{2}{|c|}{ IC 95 \% para OR } \\
& B & Sig. & OR & Inferior & Superior \\
\hline Conglomerado 2 & 1 & .000 & 1 & & \\
Conglomerado 1 & 2.225 & .000 & 9.250 & 3.297 & 25.951
\end{tabular}

En un segundo análisis se realizó una regresión logística volviendo a incluir los conglomerados 1 y 2 como predictores. Esta vez, como variable dependiente, se seleccionó el grado de implicación en los cuidados, utilizando la variable dicotomizada en los rangos baja y alta implicación en los cuidados. En este caso, se tomó como criterio para determinar una baja implicación la participación en una o ninguna de las tareas relacionadas con las AVD, y una alta implicación requería parti- cipar en dos o más (hasta seis).

En la Tabla 4 se puede ver que el grupo de personas pertenecientes al conglomerado 1 mostró una participación en los cuidados 4.125 más baja que las personas agrupadas en el conglomerado $2(\mathrm{OR}=4.125 ; \mathrm{p}<.001)$. Al nivel de confianza del $95 \%$, la OR de este factor para el modelo propuesto osciló entre 1.905 y 8.930 por lo que la asociación resultó significativa (el valor 1 no forma parte del intervalo).

Tabla 4

Modelo predictivo del grado de implicación en los cuidados a través de una regresión logística.

\begin{tabular}{c|c|c|c|c|c|} 
& & & & \multicolumn{3}{|c|}{ IC 95 \% para OR } \\
& B & Sig. & OR & Inferior & Superior \\
\hline Conglomerado 2 & 1 & .000 & 1 & & \\
Conglomerado 1 & 1.417 & .000 & 4.125 & 1.905 & 8.930
\end{tabular}

\section{Discusión}

Este estudio ha tratado de aportar información que ayude a comprender la complejidad que entraña la prestación de cuidados a personas dependientes con EP. En primer lugar, mediante el análisis de conglomerados, se crearon dos grupos de personas con perfiles definidos en base a las puntuaciones obtenidas en las escalas de personalidad y estrategias de afrontamiento. A tales grupos se les atribuye sendos estilos para los cuidados. A continuación, se exploraron las diferencias entre ambos grupos con respecto al grado de impli- cación en los cuidados y el malestar psicológico, y se encontró que uno de ellos se caracterizaba por un estilo más eficiente para hacer frente a los cuidados. De este modo, los resultados informaron que el grupo de personas con puntuaciones por debajo de la media en neuroticismo y por encima de la media en los demás factores de personalidad (extraversión; apertura; amabilidad y responsabilidad) y las estrategias de afrontamiento (planificación; afrontamiento activo; apoyo emocional; apoyo social; reevaluación positiva y aceptación) se relacionaban con una mayor implicación en los cuidados y un menor riesgo de 
padecer malestar psicológico.

Estos resultados guardan similitud con lo recogido en otras publicaciones que estudiaron estas variables. De este modo, la falta de estrategias de afrontamiento adecuadas para hacer frente a las situaciones estresantes relacionadas con los cuidados podría tener consecuencias negativas sobre el cuidador (Martínez, Colmenero y Peláez, 2002; Zambrano y Ceballos, 2007); en cambio, la personalidad vendría a actuar como un factor de protección para reducir su incidencia (Greenwell, Gray, Wersch y Schaik, 2015; Zambrano y Ceballos, 2007). Dentro de la personalidad, se ha atribuido una relación inversa entre el neuroticismo y el bienestar psicológico y una relación directa con los restantes factores de personalidad (Almagiá, Quevedo y Díaz, 2019). En este sentido, ya se había sugerido que los niveles altos de neuroticismo podían interferir a la hora de asumir un mayor número de tareas o manejar situaciones estresantes en cuidadores de personas con EP (Tew et al., 2013). En relación con las estrategias de afrontamiento, García, Manquián y Rivas (2016) encontraron una correlación significativa entre planificación y aceptación con el bienestar psicológico. Por su parte, se ha relacionado el afrontamiento por evitación con una mayor carga subjetiva y el afrontamiento centrado en las emociones con una carga subjetiva menor (Pérez-Cruz, Muñoz-Martínez, Parra-Anguita y del-Pino-Casado, 2017).

Estos datos son importantes y contribuyen a un avance en el conocimiento que se tiene en este campo, pero se plantean preguntas sobre las diferencias en el modo en que los cuidadores hacen frente a las situaciones estresantes y su participación en el cuidado a personas con EP. Determinar las necesidades reales de las personas dependientes puede ayudar a concretar, de una manera más precisa, los apoyos por parte de los cuidadores. De ahí la importancia de contar con medidas validadas que evalúen el tipo y alcance de la implicación del cuidador. El presente estudio propor- ciona un análisis conjunto de la relación entre la personalidad, las estrategias de afrontamiento, el grado de implicación del cuidador y el malestar psicológico. Este planteamiento puede contribuir a que se profundice en la investigación de perfiles de cuidadores que permitan definir estilos de cuidado más eficientes, relacionados con una mayor implicación en los cuidados y un menor impacto sobre la salud y la calidad de vida del cuidador.

Los resultados precedentes no hacen más que corroborar la importancia que se le ha dado a la atención del entorno familiar, dentro de un enfoque integral en el abordaje de la enfermedad, que no se centre solo en la persona con EP (Olsson et al., 2016). El cuidador tiene que hacer cambios en su vida cotidiana, por lo que debe asumir de manera correcta el rol de cuidador, para lo cual es necesario adquirir las competencias necesarias para la atención a la demanda de cuidados, que pueden ser proporcionadas mediante intervenciones psicosociales (Ahmad et al., 2018; Martínez, Colmenero y Peláez, 2002; Parekh et al., 2016; Ramos y Castillo 2017).

Este estudio ha permitido extraer sus conclusiones a partir de un grupo de personas cuidadoras, de las cuales el $74 \%$ eran mujeres. En las publicaciones existentes suele ser habitual encontrar este tipo de diferencias (Cueto y Martínez, 2017; Garzón Patterson, Pascual Cuesta y Collazo Lemus, 2018; Giraldo, Zuluaga y Uribe, 2018). Sin embargo, este desequilibrio en la composición de la muestra supone una limitación al estudio, por cuanto se han introducido variables como la personalidad o estrategias de afrontamiento, en las que ya de por si existen diferencias entre ambos sexos (Guadarrama, Carmona, Mendoza, López y Gutiérrez, 2018; Paez y Rovella, 2019; Vargas, Duitama y Camargo, 2014; Zentner Zúñiga y Sánchez Callalli, 2017). Esto permite planear futuras intervenciones con una perspectiva de género.

Finalmente, el malestar psicológico del cuidador puede suponer un problema para el futuro de los cuidados y los resultados obte- 
nidos en este estudio sugieren que tanto las estrategias de afrontamiento como la personalidad pueden desempeñar un papel destacado. Los aspectos psicológicos estudiados se convierten en una parte importante respecto del desarrollo de los cuidados, que son relevantes dentro del contexto en el que se desarrollan; resulta importante la incorporación de otros aspectos sociodemográficos y de la salud. Este estudio, al ser transversal, no permite determinar la contribución de otras variables que no han sido analizadas. Por ello, se proponen líneas de investigación futuras basadas en estudios cuantitativos que permitan extraer conclusiones con respecto al impacto sobre el grado de implicación y el malestar psicológico del cuidador, lo que podría contribuir a identificar estilos para los cuidados más eficientes.

\section{Referencias}

Ahmad, M., Amaya, L., Calabria, B., Arcon, A., Carrillo, D., Muños, A. S., ... y Fontalvo, D. (2018). Calidad de vida en pacientes con enfermedad de Huntington y sus cuidadores. Salud en Movimiento, 10(1), 1-15. https://core.ac.uk/ download/pdf/267928833.pdf

Almagiá, E. B. A. B., Quevedo, O. S. y Díaz, K. S. D. S. (2013). Personalidad y bienestar psicológico: un estudio en universitarios chilenos. Revista de Psicología, 9(17), 7-18. https://core.ac.uk/download/pdf/32621709.pdf

Baltar, A. L., Cerrato, I. M., de Trocóniz, M. I. F. y Márquez-González, M. (2006). Estudio e intervención sobre el malestar psicológico de los cuidadores de personas con demencia. El papel de los pensamientos disfuncionales (No. 12005). Imserso. https://www.infogerontologia.com/documents/burnout/estudiomalestarpscuidadores.pdf

Benito-León, J. (2018). Epidemiología de la enfermedad de Parkinson en España y su contextualización mundial. Revista de Neurología, 66(4), 125-134. https://doi.org/10.33588/ m.6604.2017440

Benito-León, J., Louis, E. D., Villarejo-Galende,
A., Romero, J. P. y Bermejo-Pareja, F. (2014). Under-reporting of Parkinson's disease on death certificates: A population-based study (NEDICES). Journal of the Neurological Sciences, 347(1-2), 188-192. https://doi. org/10.1016/j.jns.2014.08.048

Blanco Buitrago, S., Martínez Wilches, D., Velasco Malaver, O., Wilches Najar, M. y Bernal Jiménez, A. (2017). Riesgo de padecer enfermedades médicas asociadas a factores psicológicos en los cuidadores de pacientes diagnosticados con una enfermedad de Parkinson. Memorias de Investigación Corporación Universitaria Minuto de Dios - UNIMINUTO. http://hdl.handle.net/10656/6587

Carver, C. S. (1997). You want to measure coping but your protocol's too long: Consider the Brief COPE. International Journal of Behavioral Medicine, 4, 92-100. https://doi.org/10.1207/ s15327558ijbm0401_6

Cassaretto, M. (2010). Relación entre la personalidad y afrontamiento en adolescentes preuniversitarios. Revista Vanguardia Psicológica, 1(2), 202-225. https://dialnet.unirioja.es/ servlet $/$ articulo? codigo $=4815128$

Costa, P. y McCrae, R. (2008). NEO-FFI, Inventario NEO reducido de Cinco Factores. $3^{a} \mathrm{Ed}$. Madrid: TEA Ediciones.

Cuéllar Flores, I. y Sánchez López, M. (2012). Adaptación psicológica en personas cuidadoras de familiares dependientes. Clínica $y$ Salud, 23(2), 141-152. https://doi.org/10.5093/ cl2012a9

Cueto, B. G. y Martínez, C. G. (2017). Cuidados de enfermería al cuidador familiar de personas dependientes. RqR Enfermería Comunitaria, 5(3), 30-44. https://ria.asturias.es/RIA/ bitstream/123456789/8527/1/Archivo.pdf

Dorsey, E. R., Elbaz, A., Nichols, E., Abd-Allah, F., Abdelalim, A., Adsuar, J. C. et al. (2018). Global, regional, and national burden of Parkinson's disease, 1990-2016: a systematic analysis for the Global Burden of Disease Study 2016. The Lancet Neurology, 17(11), 939-953. https://doi.org/10.1016/S14744422(18)30295-3

Espinosa, D., Guzmán, J. A., Bautista, N. V. 


\section{Aguilar Agudo, Herruzo Cabrera y Pino Osuna}

y Ramos, J. A. (2018). Carga de trabajo del Cuidador del Adulto Mayor. Cina Research, 2(3), 28-37. https://journals.uninavarra.edu.co/index.php/cinaresearch/article/ view/135

Espinosa Miranda, K. y Jofre Aravena, V. (2012). Sobrecarga, apoyo social y autocuidado en cuidadores informales. Ciencia y Enfermería, 18(2), 23-30. https://www.redalyc.org/ pdf/3704/370441810003.pdf

Feixas, G., Evans, C., Trujillo, A., Saúl, L., Botella, L., Corbella, S. et al. (2012). La versión española del CORE-OM. Clinical Outcomes in Routine Evaluation. Outcome Measure. Revista de Psicoterapia, 23(89), 109-135. http://hdl.handle.net/2445/32884

Fernández, M. D. R., Mengíbar, E. M., Cano, M. L. y Leyva, A. F. (2016). Evaluación de los estilos de afrontamiento utilizados en cuidadores familiares de enfermos crónicos complejos. European Journal of Health Research: (EJHR), 2(3), 83-93. https://doi. org/10.30552/ejhr.v2i3.36

García, F. E., Manquián, E. y Rivas, G. (2016). Bienestar psicológico, estrategias de afrontamiento y apoyo social en cuidadores informales. Psicoperspectivas. Individuo y Sociedad, 15(3), 101-111. https://doi.org/10.5027/psicoperspectivas-Vol15-Issue3-fulltext-770

García-Ramos, R., Valdés, E. L., Ballesteros, L., Jesús, S. y Mir, P. (2016). Informe de la Fundación del Cerebro sobre el impacto social de la enfermedad de Parkinson en España. Neurología, 31(6), 401-413. https://doi.org/10.1016/j. nrl.2013.04.008

Garzón Patterson, M., Pascual Cuesta, Y. y Collazo Lemus, E. D. L. C. (2018). Relación entre depresión y características sociodemográficas en cuidadores principales de pacientes con enfermedad de Alzheimer. Revista Cubana de Enfermeria, 34(1), 114-123. http://www. revenfermeria.sld.cu/index.php/enf/article/ view/1513/330

Giraldo, D. I., Zuluaga, S. y Uribe, V. (2018). Sobrecarga en los cuidadores principales de pacientes con dependencia permanente en el ámbito ambulatorio. Medicina U.P.B, 37(2),
89-96. https://doi.org/10.18566/medupb. v37n2.a02

Gómez-Jarabo, G. y Peñalver, J. (2007). Aspectos biopsicosociales en la valoración de la dependencia. Intervención Psicosocial, 16(2), 155-173. https://doi.org/10.4321/S113205592007000200003

Greenwell, K., Gray, W., Wersch, A. y Schaik, P. (2015). Predictors of the psychosocial impact of being a carer of people living with Parkinson's disease: A systematic review. Parkinsonism and Related Disorders, 21, 1-11. https:// doi.org/10.1016/j.parkreldis.2014.10.013

Guadarrama, R. G., Carmona, V. M., Mendoza, O. M., López, M. V. y Gutiérrez, I. M. P. (2018). Diferencias en la aplicación de estrategias de afrontamiento adolescente entre alumnos hombres y mujeres del nivel medio superior. Interdisciplinaria. Revista de Psicología y Ciencias Afines, 35(1), 205-216. https://doi. org/10.16888/interd.2018.35.1.11

Hernández, J. G. E., Morales-Carmona, F., Gíaz, F. E., Pimentel, D., Meza, R. P., Henales, A. C. et al. (2006). Malestar psicológico: algunas de sus manifestaciones clínicas en la paciente gineco-obstétrica hospitalizada. Perinatología $y$ Reproducción Humana, 20(4), 112-122. https:/www.medigraphic.com/cgi-bin/new/ resumen.cgi?IDARTICULO $=13822$

Jofré, V. y Mendoza, S. (2005). Toma de decisiones en salud en mujeres cuidadoras informales. Ciencia y Enfermería, 11(1), 37-49. https://doi. org/10.4067/S0717-95532005000100005

Katz, S., Ford, A., Moskowitz, R., Jackson, B. A. y Jaffe, M. W. (1963). Studies of illness in the aged. The Index of ADL: a standarized measure of biological and psychosocial function. JAMA (Journal of American Medical Association), 185(12), 914-919. https://doi.org/10.1001/ jama.1963.03060120024016

Lazarus, R. (1993). From psychological stress to the emotions: a history of changing outlooks. Annual Review of Psychology, 44, 1-21. https:// doi.org/10.1146/annurev.ps.44.020193.000245

León-Campos, M. O., Chonchol, A. S. y Miranda-Castillo, C. (2018). Afrontamiento, apoyo social y depresión en cuidadores informales 
y su relación con necesidades no cubiertas de personas con demencia. Ansiedad $y$ Estrés, 24(2-3), 73-80. https://doi. org/10.1016/j.anyes.2018.04.001

Ma, X., Meng, G., Tan, Y., Liu, X., Zhao, Y., Yu, J., et al. (2018). Patient and Family Caregiver's Neuroticism and Conscientiousness Personality in Relation to Quality of Life of Patient With Parkinson's Disease: A Cross-Sectional Study Neuroticism and Conscientiousness Personality in Relation to QoL of Patient With PD. Frontiers in Neurology, 9, 754. https://doi. org/10.3389/fneur.2018.00754

Martin, S. C. (2016). Relational Issues Within Couples Coping With Parkinson's Disease: Implicationsand Ideas forFamily-FocusedCare. Journal of Family Nursing, 22(2), 224-251. https://doi.org/10.1177/1074840716640605

Martínez, J. A. M., Colmenero, C. J. T. y Peláez, E. M. P. (2002). Comparación entre distintas clasificaciones de las estrategias de afrontamiento en cuidadores de enfermos de Alzheimer. Psicothema, 14(3), 558-563. http:// www.psicothema.es/pdf/765.pdf

Martínez Ortega, Y. (2017). Rasgos de personalidad y estrategias de afrontamiento en personas con psicopatología. (Tesis doctoral). Universitat Autònoma de Barcelona, Barcelona, España. https://hdl.handle.net/10803/458028

Morán, C., Landero, R. y González, M. (2010). COPE-28: un análisis psicométrico de la versión en español del Brief COPE. Universitas Psychologica, 9(2), 543-552. http://www. scielo.org.co/pdf/rups/v9n2/v9n2a20.pdf?

Olsson, Y., Claren, L., Alvariza, A., Arestedt, K. y Hagell, P. (2016). Health and Social Service Access Among Family Caregivers of People with Parkinson's Disease. Journal of Parkinson's Disease, 6(3), 581-587. https://doi. org/10.3233/JPD-160811

Paez, A. E. y Rovella, A. (2019). Vínculo de apego, estilos parentales y empatía en adolescentes. Interdisciplinaria. Revista de Psicología y Ciencias Afines, 36(2), 22-38. https:// doi.org/10.16888/interd.2019.36.2.2

Parekh, N., Shah, S., McMaster, K., Speziale, A., Yun, L., Nguyen, D. et al. (2016). Effects of caregiver burden on quality of life and coping strategies utilized by caregivers of adult patients with inflammatory bowel disease. Annals of Gastroenterology, 29, 1-7. https:// doi.org/10.20524/aog.2016.0084

Pérez-Cruz, M., Muñoz-Martínez, M. A., Parra-Anguita, L. y del-Pino-Casado, R. (2017). Afrontamiento y carga subjetiva en cuidadores primarios de adultos mayores dependientes de Andalucía, España. Atención Primaria, 49(7), 381-388.

Ramos, E. C. y Castillo, V. A. M. (2017). Prevalencia del síndrome de sobrecarga y sintomatología ansiosa depresiva en el cuidador del adulto mayor. Psicología y Salud, 27(1), 53-59.

Reyes, C. A. y Muñoz-Quezada, M. T. (2019). Calidad de vida y sobrecarga en cuidadores de escolares con discapacidad intelectual. Interdisciplinaria. Revista de Psicología y Ciencias Afines, 36(1), 257-272. https://doi. org/10.16888/interd.2019.36.1.17

Santangelo, G., Garramone, F., Baiano, C., D’Iorio, A., Piscopo, F., Raimo, S. y Vitale, C. (2018). Personality and Parkinson's disease: a meta-analysis. Parkinsonism and Related Disorders, 49, 67-74. https://doi.org/10.1016/j. parkreldis.2018.01.013

Stebbins, P. y Pakenham, K. I. (2001). Irrational schematic beliefs and psychological distress in caregivers of people with traumatic brain injury. Rehabilitation Psychology, 46(2), 178. https://doi.org/10.1037/0090-5550.46.2.178

Sturm, D., Folkerts, A. y Kalbe, E. (2018). Easing Burden and Stress: Intervention Needs of Family Members of Patients with Parkinson's Disease. Journal of Parkinson's Disease, 1-7. https://doi.org/10.3233/JPD-181456

Tew, E., Naismith, S., Pereira, M. y Lewis, S. (2013). Quality of Life in Parkinson's Disease Caregivers: The Contribution of Personality Traits. Hindawi Publishing Corporation BioMed Research International, Article ID 151872. https://doi.org/10.1155/2013/151872

Tysnes, O. B. y Storstein, A. (2017). Epidemiology of Parkinson's disease. Journal of Neural Transmission, 124(8), 901-905. https:// doi.org/10.1007/s00702-017-1686-y 
Vargas, H. S., Duitama, A. G. y Camargo, Y. S. (2014). Relación entre estilos de personalidad y estrategias de afrontamiento en estudiantes de psicología. Revista Médicas UIS, 27(2). https://doi.org/10.18273/revmed

Zambrano, R. y Ceballos, P. (2007). Síndrome de carga del cuidador. Revista Colombiana de Psiquiatría, 36, 26-39. http://ref.scielo.org/ j3sqt4

Zentner Zúñiga, W. P. y Sánchez Callalli, J. (2017). Estilos de personalidad y niveles de estrés en familiares cuidadores de pacientes con trastornos psiquiátricos atendidos en el Hospital de Salud Mental "San Juan Pablo II" Cusco-2017. http://repositorio.uandina.edu.pe/ handle/UAC/1023

Recibido: 23 de abril de 2020 Aceptado: 18 de agosto de 2021 